Mauro Pinheiro Rodrigues

\title{
DESIGN DE INTERAÇÃO E COMPUTAÇÃO PERVASIVA um estudo sobre mecanismos atencionais e sistemas de informação ambiente
}

Tese de Doutorado

Tese apresentada ao Programa de Pós-Graduação em Design da PUC-Rio como requisito parcial para obtenção do título de Doutor em Design.

Orientadora: Profa. Rejane Spitz

Rio de Janeiro

Agosto de 2011 
Mauro Pinheiro Rodrigues

Design de interação e computação pervasiva: um estudo sobre mecanismos atencionais e sistemas de informação ambiente

Tese apresentada ao Programa de Pós-Graduação em Design da PUC-Rio como requisito parcial para obtenção do grau de Doutor em Design. Aprovada pela Comissão Examinadora abaixo assinada.

Profa. Rejane Spitz

Orientadora

Departamento de Artes \& Design - PUC-Rio

Prof. Nilton Gonçalves Gamba Júnior Departamento de Artes \& Design - PUC-Rio

Prof. Jorge Roberto Lopes dos Santos Departamento de Artes \& Design - PUC-Rio

Prof. Rogerio José Camara Universidade de Brasília - UnB

Prof. Luciano Rogerio de Lemos Meira Universidade Federal de Pernambuco - UFPE

Profa. Denise Berruezo Portinari Coordenadora Setorial do Centro de Teologia e Ciências Humanas - PUC-Rio 
Todos os direitos reservados. É proibida a reprodução total ou parcial do trabalho sem autorização da universidade, do autor e da orientadora.

\section{Mauro Pinheiro Rodrigues}

Graduou-se em Desenho Industrial pela Escola Superior de Desenho Industrial da Universidade do Estado do Rio de Janeiro (ESDI-UERJ) em 1995. Obteve o grau de Mestre em Design pela Pontifícia Universidade Católica do Rio de Janeiro (PUC-Rio) em 2000. É professor do quadro permanente do Departamento de Desenho Industrial da Universidade Federal do Espírito Santo (UFES). Suas atividades de pesquisa concentram-se nos seguintes temas: design de interação; design da informação; arquitetura de informação; interação humano-computador; sistemas de informação ambiente, computação pervasiva; impactos sociais do uso de tecnologias computacionais.

Ficha catalográfica

Rodrigues, Mauro Pinheiro

Design de interação e computação pervasiva : um estudo sobre mecanismos atencionais e sistemas de informação ambiente / Mauro Pinheiro Rodrigues ; orientador : Rejane Spitz. - 2011.

212 f. : il. (color.) ; $30 \mathrm{~cm}$

Tese (doutorado) - Pontifícia Universidade Católica do Rio de Janeiro, Departamento de Artes e Design, 2011.

Inclui bibliografia

1. Artes e Design - Teses. 2. Design. 3. Computação pervasiva. 4. Computação ubíqua. 5. Mecanismos atencionais. 6. Sistemas de informação ambiente. 7. Design de interação. 8. Design da informação. 9. Interação humano-computador. I. Spitz, Rejane. II. Pontifícia Universidade Católica do Rio de Janeiro. Departamento de Artes e Design. III. Título. 
Para meus pais, que me ensinaram tanto, simplesmente sendo.

Tenho orgulho de ser seu filho e de ver vocês em mim.

Para Laura, amor da minha vida, companheira de todas as horas.

Sem você seria impossível. 


\section{Agradecimentos}

Aos meus colegas do Departamento de Desenho Industrial da UFES, por terem se desdobrado para cobrir minha ausência.

Aos meus alunos da UFES, que sempre renovam minha paixão pelo ensino.

Aos meus colegas da PUC-Rio, Mirella Migliari, Brenda Lucena e Delmar Galisi, companheiros nessa longa jornada.

Às minhas amigas Georgia, Thais, Laura Lessa, Mari New, Gábi e Tê. O carinho de vocês, mesmo à distância, minimizou o isolamento do doutorado.

À Lu Baptista e ao Warren, meus "tradutores" e anfitriões tão queridos.

Aos Soulsas, Sergio, Flávio, Victor, Leo, Andre e Machado, camaradas de impagáveis sessões "descarrego", e à Luciana Mendonça. Cada um a seu modo, vocês ajudaram a manter minha sanidade.

Ao meu irmão Bruno, parceiro desde antes de nascermos.

À Déborah, a Preta, irmã querida que não entende bem o que eu faço, e sempre me faz rir de mim mesmo.

À minha querida avó Elza e ao meu saudoso avó Antenor, que me acolheram anos atrás quando eu iniciava a carreira acadêmica.

À minha orientadora Rejane, que sugeriu o tema que deu origem a este trabalho. Sou grato por sua amizade e por confiar em mim mais do que eu mesmo.

À Laura, por tanta coisa, que nem cabe aqui. 


\section{Resumo}

Rodrigues, Mauro Pinheiro; Spitz, Rejane (Orientadora). Design de interação e computação pervasiva: um estudo sobre mecanismos atencionais e sistemas de informação ambiente. Rio de Janeiro, 2011. 212p. Tese de Doutorado - Departamento de Artes e Design, Pontifícia Universidade Católica do Rio de Janeiro.

A tese investiga os diferentes mecanismos atencionais envolvidos na utilização de sistemas de informação ambiente. Para tanto, conceitua e delimita a "computação pervasiva" - a partir da qual componentes computadorizados passam a compor o ambiente e os objetos do cotidiano -, e discute as conseqüências do uso desta tecnologia, no que se refere aos impactos sociais, implicações ambientais, questões de segurança e privacidade, destacando o papel do design nessa problemática. Apresenta a evolução do design de interação, explicitando sua relação com o projeto de mídias interativas. Propõe ampliar o campo de atuação do design de interação, considerando que a tela do computador deixou de ser a principal interface com o ambiente digital, e que o projeto da interação no contexto da computação pervasiva exige uma abordagem sistêmica. Apresenta o conceito de tecnologia sem estresse (calm technology), de Weiser e Brown (1996), e aprofunda a discussão iniciada por estes autores sobre a necessidade de se projetar sistemas de informação que atuem na periferia de nossa atenção, com base nos estudos da Psicologia Cognitiva sobre mecanismos atencionais. Destaca os sistemas de informação ambiente como aqueles que mais se apropriam da idéia de apresentar informações sem exigir o foco de nossa atenção. Analisa doze sistemas de informação ambiente, investigando o modo como envolvem os mecanismos atencionais. Conclui que a definição original de Weiser e Brown (1996) não é suficiente para descrever a miríade de processos envolvidos com a captação da atenção, e aponta linhas mestras para o design de sistemas de informação ambiente, de maneira a considerar a dinâmica entre os diferentes mecanismos atencionais, o contexto de uso, o grau de engajamento do usuário, a influência da memória e a capacidade de habituação aos sistemas de informação.

\section{Palavras-chave}

Computação pervasiva; computação ubíqua; mecanismos atencionais; sistemas de informação ambiente; atenção; percepção; design de interação. 


\section{Abstract}

Rodrigues, Mauro Pinheiro; Spitz, Rejane (Advisor). Interaction design and pervasive computing: a study of attentional mechanisms and ambient information systems. Rio de Janeiro, 2011. 212p. Ph.D. Thesis Departmento de Artes e Design, Pontifícia Universidade Católica do Rio de Janeiro.

This thesis investigates the different attentional mechanisms involved when using ambient information systems. To that end, it defines and delimits "pervasive computing" - when computational resources are embedded into the environment and in everyday objects - and discusses the consequences of this technology, regarding the social impacts, environmental implications, security and privacy issues, highlighting the role of design on this matter. It presents the evolution of interaction design, emphasizing its relationship with the design of interactive media. It proposes to broaden the interaction design field, considering that the computer screen is no longer the primary interface with the digital environment, and that interaction design requires a systemic approach in the context of pervasive computing. It introduces Weiser and Brown's (1996) concept of calm technology, and deepens the discussion initiated by these authors about the need for designing information systems that act on the periphery of our attention, based on Cognitive psychology studies about attentional mechanisms. It highlights ambient information systems as those which have more properly embraced the idea of presenting information without requiring the focus of our attention. It analyzes twelve ambient information systems, investigating how the attentional mechanisms are involved in their usage. It concludes that Weiser and Brown's (1996) original concept is not sufficient to describe the myriad of processes involved with our attention, and outlines guidelines for the design of ambient information systems, in order to consider the dynamics between different attentional mechanisms, the context of use, the degree of user engagement, the influence of memory and the ability to habituate to information systems.

\section{Keywords}

Pervasive computing; ubiquitous computing; ambient information systems; attentional mechanisms; attention; perception; interaction design. 


\section{Sumário}

1. Introdução 13

1.1. Motivação e objetivo da pesquisa 27

1.2. Aspectos metodológicos 30

1.3. Estrutura da tese 34

2. Ubiqüidade computacional 36

2.1. Tecnologias que desaparecem 36

2.2. Computação pervasiva 38

2.2.1. Peças do quebra-cabeças 42

2.2.2. Aplicações da computação pervasiva 53

2.2.3. Implicações da computação pervasiva 61

3. A revolução informacional e o design de interação 76

3.1. O design e sua relação com o contexto tecnológico 77

3.2. Do design de interação ao design da experiência 82

4. Tecnologia sem estresse (calm technology) 98

4.1. O que é atenção? 101

4.1.1. Mecanismos atencionais 103

4.1.2. Funções da atenção 107

4.1.3. Percepção e atenção 112

4.1.4. A influência da memória, do aprendizado e da experiência 113

4.1.5. A influência do contexto no engajamento da atenção 116

4.1.6. Atenção como um continuum 119

5. Sistemas de informação ambiente 122

5.1. Metodologia 127

5.1.1. Critérios de seleção 127

5.1.2. Método de análise 130

5.2. Descrição e análise dos sistemas 131 
5.2.1. Ambient umbrella 134

5.2.2. Aura Orb 139

5.2.3. Datafountain 143

5.2.4. Flash bag 148

5.2.5. The Good Night Lamp 151

5.2.6. The history tablecloth 155

5.2.7. Ladybag 158

5.2.8. Power Point 165

5.2.9. Thirsty light 167

5.2.10. The Whereabouts Clock 170

5.2.11. Wattson clock 173

5.2.12. Weather patterns 179

5.3. Análise e discussão 182

5.3.1. Comparação dos sistemas de informação ambiente 191

6. Considerações finais 192

6.1. Desdobramentos: passado, presente, futuro 198

7. Referências 200 


\section{Lista de figuras}

Figura 1 - Etiquetas RFID, em diversos tamanhos $\quad 48$

Figura 2 - Implante RFID de Amal Graafstra 50

Figura 3 - Porta para gatos com leitor RFID 51

Figura 4 - Fotos dos gatos passando pela porta são tiradas

e publicadas automaticamente na Internet 51

Figura 5 - Esquema de aplicação de chips RFID em passaportes 52

Figura 6 - Continuous Glucose Monitoring (CGM) 53

Figura 7 - A evolução do computador vestível de Steve Mann, desde os anos 80 até a década de 90

Figura 8 - SportSemble, projeto do MIT 55

Figura 9 - O projeto SixthSense $\quad 56$

Figura 10 - Nike + iPod 57

Figura 11 - Esquema de comunicação do sistema QuietCare $\quad 58$

Figura 12 - Telas do sistema QuietCare 58

Figura 13 - Botanicalls $\quad 60$

Figura 14 - Kickbee $\quad 61$

Figura 15 - Diagrama ontológico do design, de Gui Bonsiepe $\quad 78$

Figura 16 - As diferentes competências e disciplinas envolvidas no design da experiência 86

Figura 17 - Processos cognitivos relacionados à atenção 103

Figura 18 - Exemplos de testes de sondagem visual 111

Figura 19 - O continuum dos processos atencionais 121

Figura 20 - Dangling String $\quad 124$

Figura 21 - Ambient Umbrella 134

Figura 22 - Ambient Umbrella em uso 135

Figura 23 - Site da empresa Ambient Devices 136

Figura 24 - Análise do Ambient Umbrella 138

Figura 25 - Aura Orb 139

Figura 26 - Análise do Aura Orb, primeira etapa de uso 141

Figura 27 - Análise do Aura Orb, segunda etapa de uso 142 
Figura 28 - Datafountain

Figura 29 - Os pesquisadores envolvidos na construção

da Datafountain

Figura 30 - Análise da Datafountain 147

Figura 31 - Flash bag 148

Figura 32 - Análise do Flash bag 150

Figura 33 - The Good Night Lamp 151

Figura 34 - The Good Night Lamp 152

Figura 35 - Análise da Good Night Lamp 154

Figura 36 - The History Tablecloth 155

Figura 37 - Análise da History Tablecloth 157

Figura 38 - O protótipo da Ladybag 158

Figura 39 - Ladybag: diagrama com a localização dos sensores e as emoções correspondentes 159

Figura 40 - Ladybag como Effective Organizing System 160

Figura 41 - Análise da Ladybag, como Affective Communication System, na perspectiva do usuário

Figura 42 - Análise da Ladybag, como Affective Communication

System, na perspectiva do observador

Figura 43 - Análise da Ladybag, como Effective Organizing System 164

Figura 44 - Power Point 165

Figura 45 - Análise do Power Point 166

Figura 46 - Thirsty Light 167

Figura 47 - Análise da Thirsty Light 169

Figura 48 - The Whereabouts clock 171

Figura 49 - Análise do Whereabouts Clock 172

Figura 50 - O sistema de medição de consumo de energia Wattson 173

Figura 51 - Wattson 174

Figura 52 - Holmes 174

Figura 53 - Tela do programa Holmes 176

Figura 54 - Análise do Wattson $\quad 177$

Figura 55 - Análise do Holmes 178

Figura 56 - Weather Patterns $\quad 179$

Figura 57 - Análise do Weather Patterns 181 
Figura 58 - Ambient umbrella e Thirsty light 183

Figura 59 - Wattson e Holmes 184

Figura 60 - Comparação entre Wattson e Holmes 184

Figura 61 - Detalhe da análise do Whereabouts clock 185

Figura 62 - Versão EOS da Ladybag 187

Figura 63 - Aura Orb 188

\section{Lista de tabelas}

Tabela 1 - As principais tendências no desenvolvimento da tecnologia computacional $\quad 40$

Tabela 2 - Adaptação versus habituação 107

Tabela 3 - Teste Stroop $1 \quad 109$

Tabela 4 - Teste Stroop $2 \quad 109$

Tabela 5 - Ícones usados na classificação dos sistemas 132

Tabela 6 - Classificação dos sistemas de informação ambiente 133 